\title{
Pulmonary Alveolar Proteinosis and Tuberculosis in a Diabetic Patient: A Rare or a Seldom Diagnosed Association?
}

Pereira-Silva J.L., Marinho M.M.M.A., Veloso T.V.B. and Coelho Filho J.C.
Pulmonary Service, Professor Jorge Valente Hospital, Salvador; Pathology Service, José Silveira Foundation, Salvador, BA, Brazil

\begin{abstract}
A case of Pulmonary Alveolar Proteinosis (PAP), in association with tuberculosis, is described in a 35-year-old diabetic patient. Lung biopsy showed an intra-alveolar accumulation of PASpositive material, and multifocal granulomas compatible with tuberculosis. The bronchoalveolar culture was positive for Mycobacterium tuberculosis. PAP results from an imbalance of the mechanisms that regulate the homeostasis of the surfactant, where specific proteins are involved, especially SP-A and SP-D, the cytokines, IL-10 and GM-CSF, in addition to alveolar macrophages and type-II pneumocytes. Chemotaxis and phagocytic capacity are reduced. PAP and diabetes share several immunological disfunctions that may increase the risk for tuberculosis. Although there are no controlled studies, the diagnosis of PAP in diabetic patients with tuberculosis must be considered.
\end{abstract}

Key Words: Pulmonary Alveolar Proteinosis (PAP), tuberculosis, diabetic patient.

Pulmonary Alveolar Proteinosis (PAP) was first reported by Rosen, et al. [1] in 1958, in an attempt to characterize an abnormal accumulation of PAS-positive (periodic acid-Schiff) phospholipoprotein material in the alveoli. Two forms are described: primary or idiopathic, which occurs in the absence of another illness or a known environmental exposure; and secondary, when associated with another morbid condition, especially infectious or neoplastic, in various states of immunosuppression [2], as well as in those resulting from theinhalation of chemical agents and mineral particles(silica, aluminum, titanium, and some insecticides) [3]. Several etiological agents have been identified in this population: Aspergillus sp. [4], Nocardia sp. [5-7], Mycobacterium sp. [8-13], Cryptococcus neoformans [14], Histoplasma capsulatum [15],

Received on 17 March 2002; revised 28 June 2002.

Address for correspondence: Dr. Jorge L. Pereira-Silva. Rua Conselheiro Corrêa de Menezes, 91 - Candeal. Salvador, Bahia, Brazil. Zip code: 40.295-030. Phone/Fax: (55 71) 334-7547. Email:cpc-ba@svn.com.br.

The Brazilian Journal of Infectious Diseases 2002;6(4):188-195 (C) 2002 by The Brazilian Journal of Infectious Diseases and Contexto Publishing. All rights reserved.

$1413-8670$
Pneumocystis carinii [16], and virus [17]. Rarely, cases of PAP may be associated with Mycobacterium tuberculosis [10,13]. Witty, et al. [18] noted a high incidence (42\%) of Mycobacterium avium-intracellulare in systematic cultures of therapeutic lung lavage material from PAP patients. Several recent studies [19-32] have related PAP's pathogenesis and opportunistic infections to an imbalance of the mechanisms that regulate the homeostasis of the surfactant, where specific proteins are involved, especially SP-A and SP-D, cytokines, especially IL-10 and GM-CSF, in addition to alveolar macrophages and type-II pneumocytes.

The risk for tuberculosis in diabetic patients is greater than in the general population. Mycobacteria have been isolated from BAL of PAP carriers. It is possible that the association between these clinical entities is more than coincidental. PAP is usually a pathological diagnosis and diabetic patients with tuberculosis do not routinely undergo biopsies.

\section{Case Report}

Thirty five-year-old African-Brazilian female nurse aid, an insulin-dependent diabetic, was admitted with 
a history of persistent dry cough for 30 days, together with left sided pleuritic chest pain, mild dyspnea and fever. In addition, she complained of weakness, anorexia and unmeasured weight loss. She had been using NPH insulin (24 UI in the morning and $8 \mathrm{UI}$ at night) for the previous 9 years. At the time of admission, she was in a fair general state, thin and slightly pale. BP: 130x80 mm Hg; HR: 120bpm; RR: 24bpm; T.: $38.5^{\circ} \mathrm{C}$. Crackles were present in the lower third of the left hemithorax.

Lab tests and image diagnosis exams:

- WBC count: $12,200 / \mathrm{mm}^{3}$ (segs $68 \%$, bands $8 \%$, eos $1 \%$, baso $1 \%$, lymphs $21 \%$, monocytes $2 \%$ ).

- RBC count: 3.67 million $/ \mathrm{mm}^{3}$. Hematocrit 29.4\%. Hemoglobin 10g\%. Platelets $545,000 / \mathrm{mm}^{3}$.

- Glucose: $223 \mathrm{mg} / \mathrm{dL}$

- Urea: $22 \mathrm{mg} / \mathrm{dL}$. Creatinine: $0.8 \mathrm{mg} / \mathrm{dL}$

- $\mathrm{Na}: 136 \mathrm{mEq} / \mathrm{L} ; \mathrm{K}: 4,7 \mathrm{mEq} / \mathrm{L} ; \mathrm{Ca}: 9.7 \mathrm{mg} /$ $\mathrm{dL} ; \mathrm{Mg}: 1.6 \mathrm{mg} / \mathrm{dL}$.

- AFB's (3 negative samples)

- PPD: $15 \mathrm{~mm}$

- AST: 44UI/L.ALT: $13 \mathrm{UI} / \mathrm{L}$

- Total bilirubin: 0.4 (direct: 0.1$)$

- Prothrombin time: 68\% (13.3" INR: 1.24). APTT: 32".

- $\mathrm{ABG}$ 's (FI02=0,21): $\mathrm{pH}:$ 7.4; $\mathrm{PaO}_{2}: 76.5 \mathrm{mmHg} ; \mathrm{PaCO}_{2}: 40.4 \mathrm{mmHg}$; $\mathrm{HCO}_{3}: 25.1 \mathrm{mEq} / \mathrm{L} ; \mathrm{SatO}_{2}: 95.3 \%$.

- Serology for HIV: negative.

- Chest X-rays: non-homogeneous areas of alveolar consolidation on the left lower lobe (Figure 1).

- High-resolution computed tomography (Figure 2): confluent alveolar nodules, areas of multifocal consolidation and a tree-inbud pattern.

Evolution. Fiberoptic bronchoscopy with bronchoalveolar lavage (BAL) and trans-bronchial biopsies (TBB) was performed on the left lower lobe. Direct exams for bacteria, fungi and AFB in the BAL were negative. The TBB showed non-specific inflammatory changes. A left sided pneumothorax developed as a complication of bronchoscopy, requiring close chest tube drainage. AFB was recovered from the pleural fluid and the fourth sputum sample. Thoracoscopy was performed due to a lack of lung expansion and a persistent air leak. An open biopsy was then obtained as well as a new sputum exam. AFB was again recovered from the pleural surface and from the sputum $\left(5^{\text {th }}\right.$ sample). Isoniazid, rifampicin and pyrazinamide were instituted. A histopathological exam (Figure 3.) revealed lung tissue with altered structure, multiple granulomatous reaction foci and central caseous necrosis, epithelioid histiocytes and giant multinucleus cells, in addition to some lymphocytes. The direct exam for fungus was negative. The AFB smear was positive. In the remaining lung tissue, alveoli were filled with acidophilus material and macrophages. This intra-alveolar substance stained positively with PAS (Figure 4.). The BALculture showed Mycobacterium tuberculosis. These findings supported the diagnosis of pulmonary tuberculosis and PAP(Figures 4 and 5). She was discharged from the hospital, with a recommendation for ambulatory follow-up.

\section{Discussion}

The incidence of PAP in the general population is 1 in 2 million people [26] with a 3 to 1 male-to-female predominance. Almost $80 \%$ of the patients are between 20 and 50 years old, although it has also been described in newborns [33,34], school age children [35,36], and in the elderly [37]. The main symptom is exertion dyspnea. Many patients present with dry cough or with opalescent and viscous sputum. Asthenia and weight loss may be present. Fever is more common in the secondary form. Chest pain and hemoptysis are not frequent in the primary form. The physical examination is nonspecific, with predomination of crackles in the affected areas [3,26]. The most common X-ray pattern is a symmetrical, bilateral, alveolar infiltrate, predominantly in the lower lobes. In some cases, the infiltrate is focal and asymmetric. There is usually no pleural effusion, mediastinal or pulmonary 
Figure 1. Chest X-ray: non-homogeneous areas of alveolar consolidation on the left lower lobe

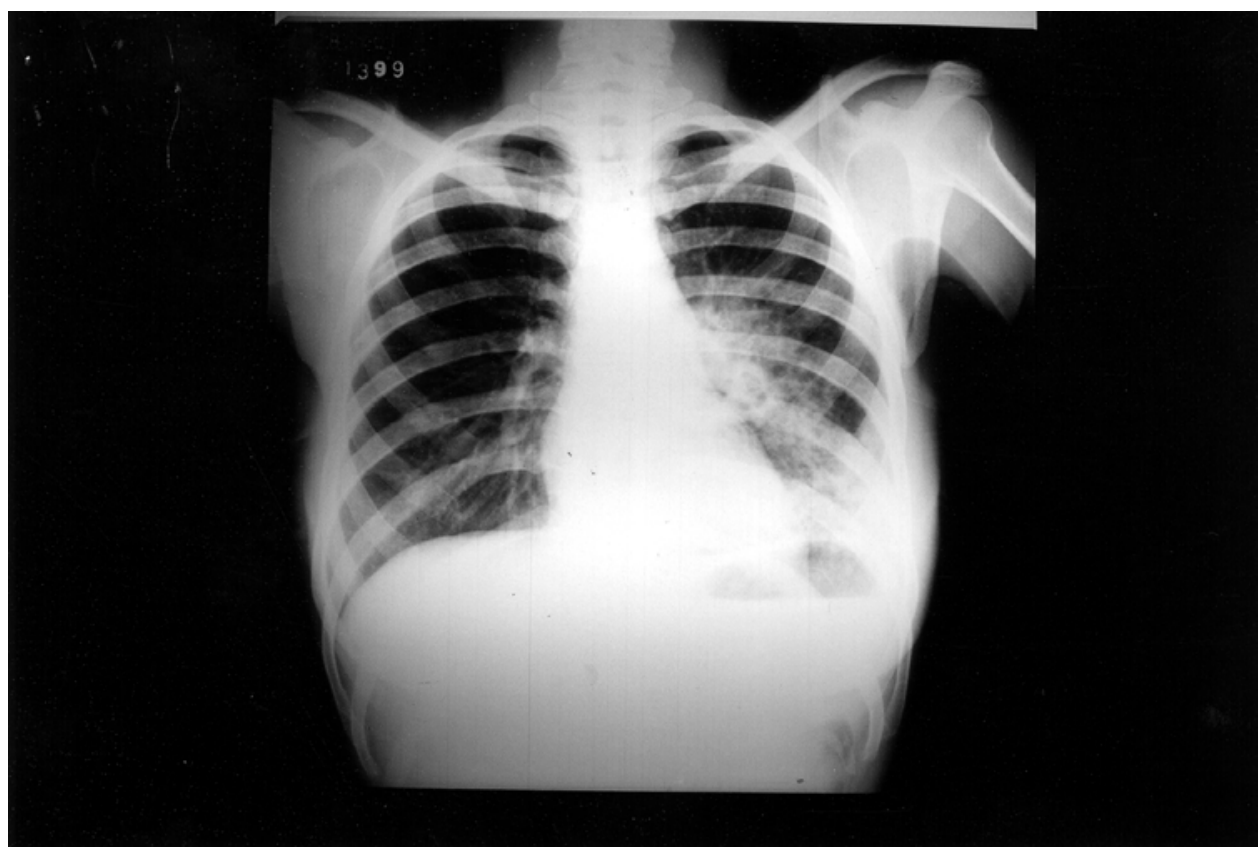

Figure 2. High-resolution computed tomography: confluent alveolar nodules, areas of multifocal consolidation and a tree-in-bud pattern

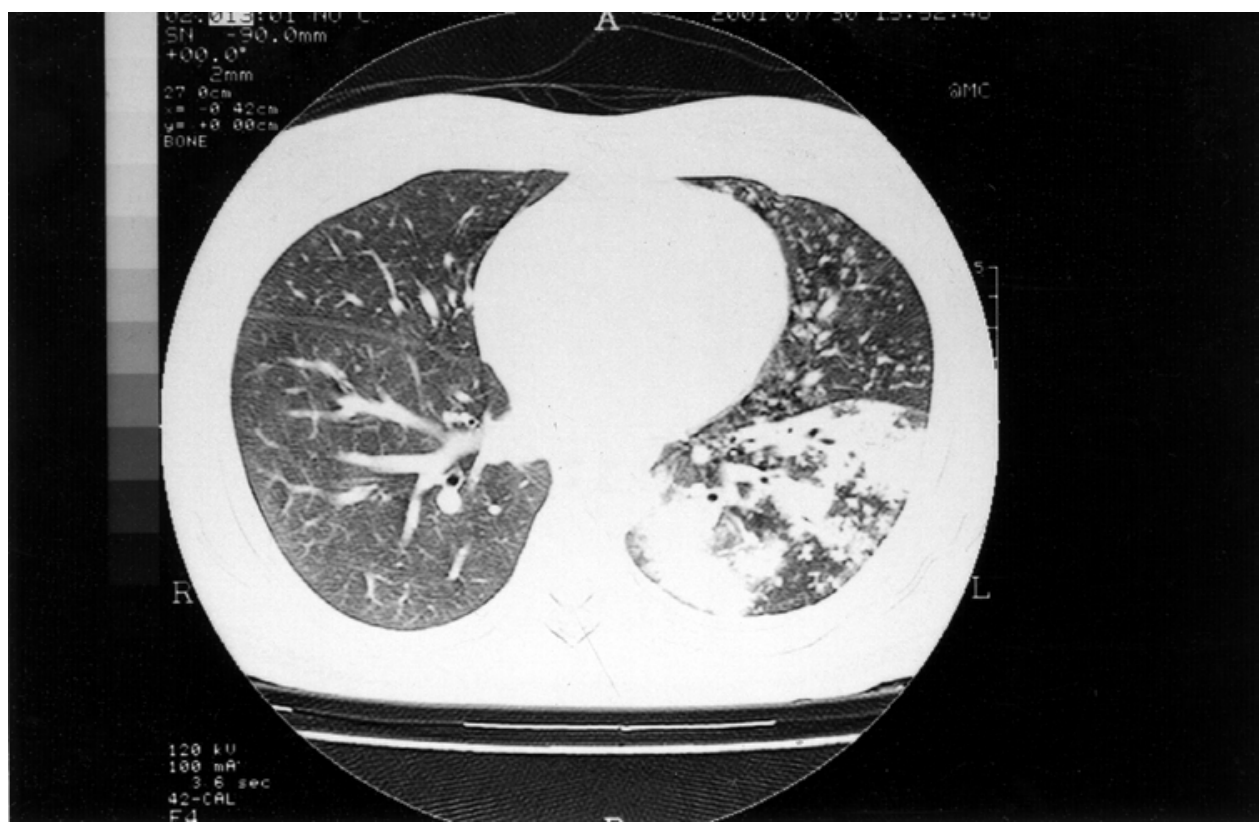


Figure 3. Histopathological exam (H.E.): lung tissue with an altered structure, with multiple granulomatous reaction foci and central caseous necrosis, epithelioid histiocytes and giant multinucleus cells, in addition to some lymphocytes. In the remaining lung tissue, the alveoli were filled with acidophilus material and macrophages

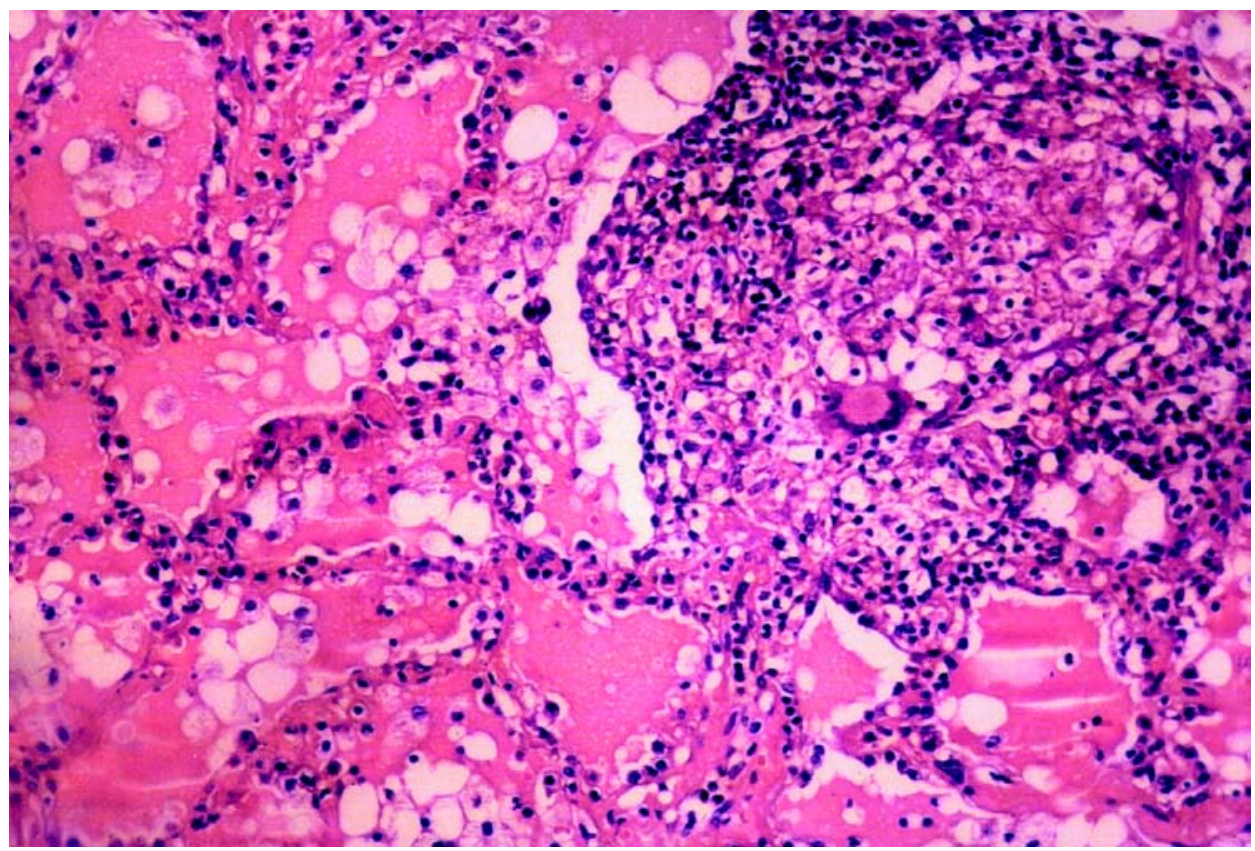

Figure 4. Periodic acid-Schiff stain (PAS): the intra-alveolar substance stained positively with PAS

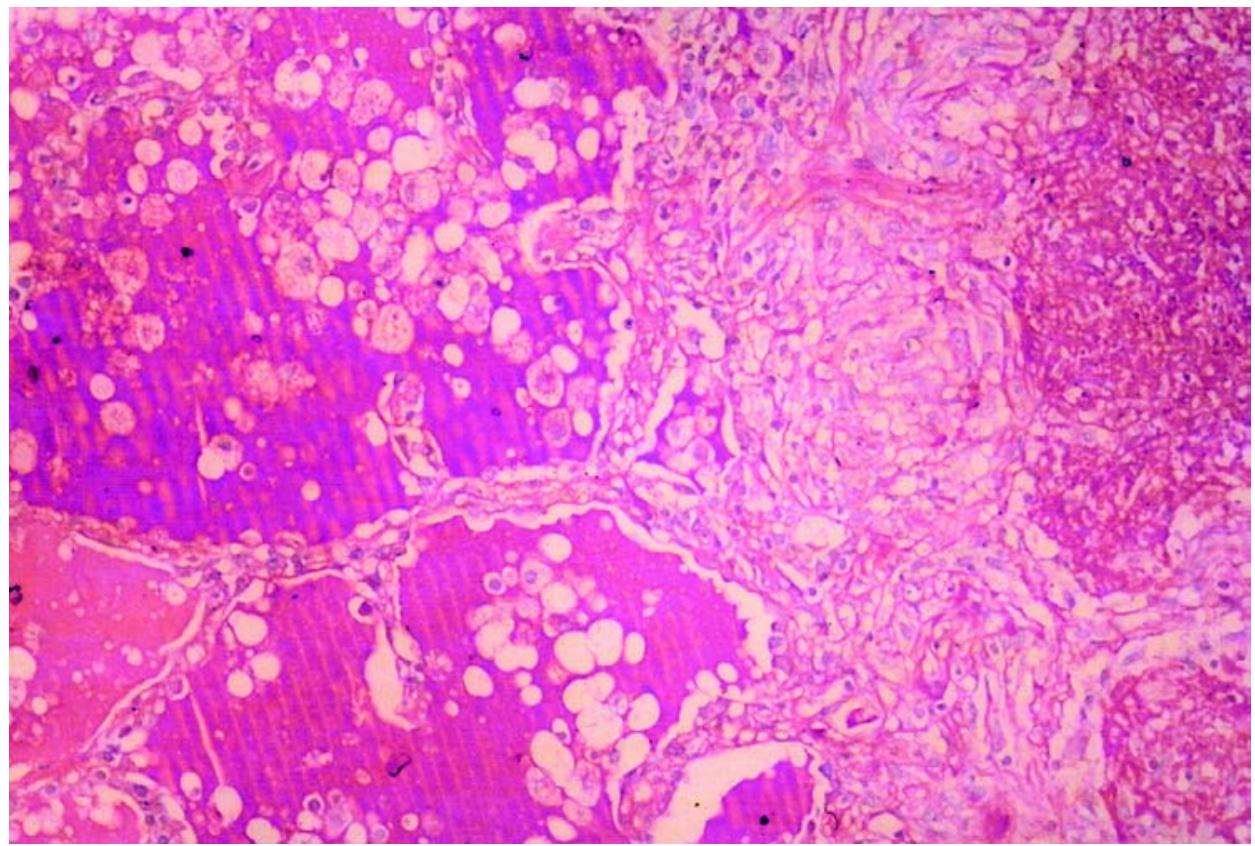


adenopathies. The high-resolution computed tomography usually shows coalescent alveolar nodules, sometimes evolving into areas of multifocal consolidation, resulting from a complete filling of alveolar spaces and zones of increasing attenuation with the ground-glass pattern, which results from partial filling of alveolar structures with the proteinaceous material. At times, ground-glass areas are delimited by zones of normal parenchyma that characterize the geographic distribution. The presence of intra-alveolar and interlobular septa inside the ground-glass zones characterizes the crazy paving pattern that, although very common in PAP, is not specific, and may be observed in Pneumocystis carinii pneumonia and in cytomegalovirus infections [38].

An elevated LDH is sometimes seen [3]. The predominant functional abnormality is a restrictive ventilatory disturbance, with a reduction of lung volumes and diffusion capacity [39]. Hypoxemia and an increase of D(A-a $0_{2}$, aggravated by exercise, are proportional to the level of lung involvement. These alterations result from a right-to-left shunt, where lung capillaries perfuse poorly ventilated alveolar units, filled with the lipoprotein material [39]. Pulmonary function tests are useful to evaluate the illness' severity, its progress and its response to therapy. Fiberoptic bronchoscopy is a routine procedure, due to its capacity for ruling out other etiologies and because it can demonstrate the characteristic granular, amorphous, PAS-positive lipoprotein material in the bronchoalveolar fluid [3]. Although the histopathological exam of the lung tissue, obtained through thoracotomy or transbronchial biopsy, is the gold-standard, PAP diagnosis can be consistently based on clinical-radiological findings plus bronchoalveolar lavage, as long as other diagnoses can be excluded [3]. The most effective treatment is a whole-lung lavage, although in up to $25 \%$ of the cases there will be spontaneous resolution [40]. This procedure is reserved for those whose daily activities are limited due to dyspnea, especially if $\mathrm{Pa}_{2}<70 \mathrm{mmHg}$ or D(A-a) $0_{2}>40 \mathrm{mmHg}$, causing greater discomfort and significant probability of disease progress [41].

PAP pathogenesis seems to be associated with an excessive secretion of surfactant and alteration of its quality. Besides reducing the surface tension of the alveoli, the surfactant, composed of $90 \%$ lipids and $10 \%$ proteins, plays an important role in the immunological mechanisms of lung natural defense and possibly other mucous surfaces [22]. The homeostasis of the surfactant is a result of a complex, dynamic process, involving alveolar macrophages and type-II pneumocytes, with active participation in collection, degradation and recycling. The lipoprotein material that results from lung lavage in PAP patients is made up of a phospholipid fraction - of which lecithin is the main component plasmatic proteins and proteins specific to the surfactant. The hydrophobic proteins (SP-B and SP-C) and hydrophilic proteins (SP-A and SP-D), components of the surfactant, are synthesized and secreted mainly by the type-II pneumocytes [22,26]. A recent analysis relates SP-B deficiency to PAP's pathogenesis, which results from mutations of the SP-B gene. The mutation 121 ins 2 is present in almost $2 / 3$ of individuals with this deficiency [42]. SP-A and SP-D play an important role in the organism's defense mechanism. They bind to the cellular wall lipopolysaccharides of various pathogens, modulating the phagocytic activity of the macrophages $[23,27]$. In addition, the lipoprotein material has the capacity of neutralizing the oxidizing stress resulting from the phagocytic process of the macrophages. As was demonstrated, the incubation of Mycobacterium tuberculosis with SP-D results in bacillus agglutination. On the other hand, SP-D binds itself minimally on nonvirulent Mycobacterium smegmatis [19]. In the first stage of infection by Mycobacterium tuberculosis, the bacilli that reach the alveoli are phagocytized by macrophages through the immunomodulation of the SPA and SP-D [20,21]. In PAP, chemotaxis and the phagocytic capacity of the macrophages are affected. This defect seems to be acquired, because after incubation with fluid obtained from lung lavage of PAP patients normal macrophages present a diminished phagocytic capacity [26]. Besides, therapeutic lung lavage usually reestablishes the macrophagic function and decreases the incidence of opportunistic infections [25]. On the other hand, in some cases, treatment for tuberculosis may cause the PAP to partially or totally subside [24]. 
In diabetic individuals, especially when poorly controlled, the functional damage to the polymorphonuclear neutrophils (adherence, chemotaxis and bactericidal activity), together with a dysfunction of the monocytic-macrophagic system and of cellular immunity, determine a greater risk of infections [43]. In these individuals the risk for tuberculosis is 2.0 to 3.6 times greater than in the general population [43]. However, there are no controlled studies that show a higher tendency of opportunistic infections in diabetic patients with PAP. A recent study [28], in which the concentration of the surfactant apoprotein (SP-A) was measured in the amniotic liquid of pregnant diabetics $(n=29)$ and non-diabetic pregnant women $(n=358)$, revealed that its level is directly proportional to the time of pregnancy (less than $3 \mathrm{mg} / \mathrm{mL}$ between the $30^{\text {th }}$ and the $31^{\text {st }}$ week, and $24 \mathrm{mg} / \mathrm{mL}$ between the $40^{\text {th }}$ and the $41^{\text {st }}$ week). In pregnant diabetic women, this concentration is reduced.

It has been shown that laboratory rats with deficiency of the gene that codifies the GM-CSF produce an abnormal accumulation of surfactant, similar to that seen in humans with PAP [29]. Experimental models, designed primarily for the study of hematopoiesis in rats, suggest that mutations of the gene that codifies GM-CSF [42] or of the beta subunit of its receptor (I13rb1) [44] may stimulate metabolic alterations in the surfactant, which are responsible for PAP [31,42]. There is incipient evidence that seems to demonstrate that daily replacement of GM-CSF (3$9 \mu \mathrm{g} / \mathrm{kg} /$ day) during 12 weeks, in PAP patients, stimulates clinical, radiological and functional improvement, without repercussions in the WBC count [45]. In this study, the therapeutic response occurred between the $8^{\text {th }}$ and the $12^{\text {th }}$ week [45].

Kitamura, et al. [46] developed an agglutination test on latex for serological diagnosis of primary PAP, with $100 \%$ sensitivity and $98 \%$ specificity, based on the identification of antibodies against GM-CSF in the BAL fluid of individuals with PAP.

These observations regarding PAP pathogenesis open new horizons for diagnosis and future therapeutic resources. The systemic immunological disturbances that take place in diabetic patients, along with focal lung alterations that turn PAP carriers more vulnerable to respiratory infections - among them tuberculosis point towards a possible association.

\section{Conclusion}

Pulmonary Alveolar Proteinosis results from an abnormal accumulation of a phospholipoprotein substance in the alveoli. It is called primary or idiopathic when no other morbid condition or known environmental exposure is present. In its secondary form, it is associated with respiratory infections by various etiologic agents, myeloproliferative illnesses, various states of immunosuppression, or environmental exposure to chemical agents or inorganic particles. Rarely, it may be associated with tuberculosis. The predominant functional alteration is restrictive ventilatory disturbance, with a reduction of lung volumes and diffusion capacity. Hypoxemia and an increase of $\mathrm{D}(\mathrm{A}-\mathrm{a}) \mathrm{O}_{2}$, aggravated by exercise, result from perfusion of poorly ventilated areas. Although the histopathological exam is the goldstandard, the diagnosis can be based on suggestive findings from high-resolution computed tomography, and on characteristic aspects of the bronchoalveolar lavage. Whole-lung lavage in selected cases is the most effective treatment. New concepts regarding its pathogenesis, where a disturbance is described in the surfactant's homeostasis, involving proteins (SP-A and SP-D), cellular elements (alveolar macrophages and type-II pneumocytes) and an imbalance of the activity of cytokines (IL-10 and GM-CSF), may offer new opportunities for the diagnosis and treatment of Pulmonary Alveolar Proteinosis.

The role of diabetes, causing a greater susceptibility to tuberculosis in PAP patients, is not well known. However, considering the various immunological disturbances that are common to these entities, the possibility of a diagnosis of PAP in diabetic patients suffering from pulmonary tuberculosis must be considered. 


\section{Acknowledgement}

We are indebted to Octavio H. C. Messeder, M.D. for reviewing this manuscript.

\section{References:}

1. Rosen S.H., Castleman B., Liebow A.A. Pulmonary alveolar proteinosis. N Engl J Med 1958;258:1123-42.

2. Ruben F.L., Talamo T.S. Secondary pulmonary alveolar proteinosis occurring in two patients with acquired immune deficiency syndrome. Am J Med 1986;80:1187-90.

3. Wang B.M., Stern E.J., Schmidt R.A., Pierson D.J. Diagnosing pulmonary alveolar proteinosis. A review and an update. Chest 1997;111:460-6.

4. Wuhrmann F., Mark G.J., Wick A., et al. Alveolar pulmonary proteinosis and aspergillosis with reactive reticulosis following silage work. A contribution on health hazards in agricultural work. Schweitz Med Wochenschr 1965;95:1738-44.

5. Burbank B., Marrione T.G., Cutler S.S. Pulmonary alveolar proteinosis and nocardiosis. Am J Med 1960;28:1002.

6. Martinez Maldonado M., Ramirez de Arellano G. Pulmonary alveolar proteinosis, nocardiosis and chronic granulocytic leukemia. South Med J 1966;59:901-5.

7. Pascual J., Gomez Aguinaga M.A, Vidal R., et al. Alveolar proteinosis and nocardiosis: a patient treated by bronchopulmonary lavage. Postgrad Med J 1989;65:674-7.

8. Bakhos R., Gattuso P., Arcot C., Reddy V.B. Pulmonary alveolar proteinosis: an unusual association with Mycobacterium avium-intracellulare infection and lymphocitic interstitial pneumonia. South Med J 1996;89:801-2.

9. Couderc L.J., Bernaudin J.F., Epardeau B., et al. Pulmonary alveolar proteinosis and disseminated Mycobacterium avium infection. Respir Med 1996;90:641-2.

10. Lathan S.R. Jr., Williams J.D. Jr., Mclean RL., et al. Pulmonary alveolar proteinosis. treatment of a case complicated by tuberculosis. Chest 1971;59:452-4.

11. Owens M.W., Kinasewitz G.T., Gonzales E. Sandblaster's lung with mycobacterial infection. Am J Med Sci 1988;295:554-7.

12. Ramirez J. Pulmonary alveolar proteinosis. treatment in a case complicated by tuberculosis. Am Rev Respir Dis 1967;95:491-5.

13. Reyes J.M., Putong P.B. Association of pulmonary alveolar lipoproteinosis with mycobacterial infection. Am J Clin Pathol 1980;74:478-85.
14. Sunderland W.A., Campbell R.A., Edwards M.J. Pulmonary alveolar proteinosis and pulmonary cryptococcosis in an adolescent boy. J Pediatr 1972;80:450-6.

15. Hartung M., Salfelder K. Pulmonary alveolar proteinosis and histoplasmosis: report of three cases. Virchows Arch A Pathol Anat Histol 1975;368:281-7.

16. Tran Van Nhieu J., Vojtck A-M., Bernaudin J-F., et al. Pulmonary alveolar proteinosis associated with Pneumocystis carinii. Ultrastructural identification in bronchoalveolar lavage in AIDS and immunocompromised non-AIDS pattients. Chest 1990;98:801-5.

17. Ranchod M., Bissell M. Pulmonary alveolar proteinosis and cytomegalovirus infection. Arch Pathol Lab Med 1979;103:139-42.

18. Witty L.A., Tapson V.F., Piantadosi C.A. Isolation of mycobacteria in patients with pulmonary alveolar proteinosis. Medicine (Baltimore) 1994;73:103-9.

19. Fergusson J.S., Voelker D.R., McCormack F.X., Schlesinger L.S. Surfactant protein D binds to Mycobacterium tuberculosis bacilli and lipoarabinomannan via carbohydrate-lecitin interations resulting in reduced phagocytosis of the bacteria by macrophages. J Immunol 1999; 163:312-21.

20. Floros J, Lin H.M., Garcia A., et al. Surfactant protein genetic marker alleles identify a subgroup of tuberculosis in a mexican population. J Infect Dis 2000; $182: 1473-8$.

21. Gaynor C.D., McCormack F.X., Voelker D.R., et al. Pulmonary surfactant protein A mediates enhanced phagocytosis of Mycobacterium tuberculosis by a direct interation with human macrophages. J Immunol 1995; 155:5343-51.

22. Haagsman H.P., Diemel R.V. Surfactant-associated proteins: functions and structural variation. Com Biochem Physiol A Mol Integr Physiol 2001;129:91-108.

23. Hickman-Davis J.M., Fang F.C., Nathan C., et al. Lung surfactant and reactive oxygen-nitrogen species: antimicrobial activity and host-pathogen interactions. Am J Physiol Lung Cell Mol Physiol 2001;281:L517-32.

24. Morinari H., Terashi R., Okubo S., et al. Remission of pulmonary alveolar proteinosis during antituberculous chemotherapy. Eur J Respir Dis 1987;71:54-5.

25. Okano A., Sato A., Chida K., et al. Improvement in alveolar macrophage function after terapeutic lung lavage in pulmonary alveolar proteinosis. Nihon Kyobu Shikkan Gakkai Zasshi 1990;28:723-8.

26. Shah P.L., Hansell D., Lawson P.R., et al. Pulmonary alveolar proteinosis: clinical aspects and concepts on pathogenesis. Thorax 2000;55:67-77. 
27. Sidobre S., Nigou J., Puzo G., Riviere M. Lipoglycans are putative ligands for the human pulmonary surfactant protein A attachment to mycobacteria. Critical role of the lipids for lecitin-carbohydrate recognition. J Biol Chem 2000;275:2415-22.

28. Snyder J.M., Kwun J.E., O’Brien J.A., Rosenfeld C.R., Odom M.J. The concentration of the $35-\mathrm{kDa}$ surfactant apoprotein in amniotic fluid from normal and diabetic pregnancies. Pediatric Res 1988;24:728-34.

29. Stanley E., Lieschke G.J., Grail D., et al. Granulocyte/ macrophage colony-stimulating factor-deficient mice show no major pertubation of hematopoiesis but develop a characteristic pulmonary pathology. Proc Natl Acad Sci USA 1994;91:5592-6.

30. Thomassen M.J., Yi T., Raychaudhuri B., et al. Pulmonary alveolar proteinosis is a disease of decreased availability of GM-CSF rather than an intrinsec cellular defect. Clin Immunol 2000;95:85-92.

31. Tchou Wong K.M., Harkin T.J., Chi C., et al. GM-CSF gene expression is normal but protein release is absent in a patient with pulmonary alveolar proteinosis. Am J Respir Crit Care Med 1997;156:1999-2002.

32. Mildenberger E., deMello D.E., Lin Z., et al. Focal congenital alveolar proteinosis associated with abnormal surfactant protein B messenger RNA. Chest 2001;119:645-7.

33. Coleman M., Dehner L.P., Sibley R.K., et al. Pulmonary alveolar proteinosis: an uncommon cause of chronic neonatal respiratory distress. Am Rev Respir Dis 1980;121:583-6.

34. Mahut B., Delacourt C., Scheinmann P., et al. Pulmonary alveolar proteinosis: experience with eight pediatric cases and a review. Pediatrics 1996;97:117-22.

35. Colon A.R. Jr., Lawrence R.D., Mills S.D., et al. Childhood pulmonary alveolar proteinosis (PAP). Report of a case and review of the literature. Am $J$ Dis Child 1971; 121:481-5.

36. Wilkinson R.H., Blanc W.A., Hagstrom J.W. Pulmonary alveolar proteinosis in three infants. Pediatrics 1968; $41: 510-5$.

37. Prakash U.B., Barham S.S., Carpenter H.A., et al. Pulmonary alveolar phospholipoproteinosis: experience with 34 cases and review. Mayo Clin Proc 1987;62:499-518.

38. Murch C.R., Carr D.H. Computed tomography appearances of pulmonary alveolar proteinosis. Clin Radiol 1989; 40:240-3.

39. Rogers R.M., Levin D.C., Gray B.A., et al. Physiologic effects of bronchopulmonary lavage in alveolar proteinosis. Am Rev Respir Dis 1978;118:255-64.

40. Davidson J.M., Macleod W.M. Pulmonary alveolar proteinosis. Br J Dis Chest 1969;63:13-28.
41. Kariman K., Kylstra J.A., Spock A. Pulmonary alveolar proteinosis: prospective clinical experience in 23 patients for 15 Years. Lung 1984; 162:223-31.

42. DeMello D.E., Lin Z. Pulmonary alveolar proteinosis: a review. Pediatr Pathol Mol 2001;20:413-32.

43. Sentochnik D.E., Eliopoulos G.M. Infection and Diabetes. In: Kahn C.R., Weir G.C.eds. Joslin's Diabetes. $3^{\text {rd }}$ ed. Philadelphia, USA: Lea \& Febiger, 1994, pp 867-88.

44. Tredano M., Blic J.D., Griese M., et al. Clinical, biological and genetic heteroneity of the inborn errors of pulmonary surfactant metabolism: SP-B deficiency and alveolar proteinosis. Ann Biol Clin (Paris) 2001;59:131-48.

45. Kavuru M.S., Sullivan E.J., Piccin R., et al. Exogenous granulocyte-macrophage colony-stimulating factor administration for pulmonary alveolar proteinosis. Am J Respir Crit Care Med 2000;161:1143-8.

46. Kitamura T., Uchida K., Tanaka N., et al. Serological diagnosis of idiopathic pulmonary alveolar proteinosis. Am J Respir Crit Care Med 2000;162:658-62. 